\title{
NEW DIRECTIONS IN MULTIPHOTON DISSOCIATION
}

\author{
P. F. GONZÁLEZ-DÍAZ, M. SANTOS, C. L. SIGÜENZA \\ and J. A. TORRESANO
}

Instituto de Óptica "Daza de Valdés". C.S.I.C., Serrano 121, 28006 Madrid (Spain)

(Received 24 July 1991; in final form 15 October 1991)

\begin{abstract}
.
Unimolecular multiphoton dissociation offers us a rather complete scheme where the transition from quantum mechanics to the classical regime can be experimentally followed. In the light of this idea, a new interpretation of the unimolecular multiphoton dissociation process is presented. The role that the intramolecular generation of instabilities and chaos may play in that process is discussed, both in single and double exposure experiments. Delayed double irradiation is also discussed and a criterion to define an effective single pulse notion in that kind of experiment is given.
\end{abstract}

1. A question that may be a little worrying in any experiment where we irradiate a collection of atoms or molecules with a high density of photons is the definition of measurement. ${ }^{1}$ Actually, most measurements use radiation as a probe to check the changes which have occurred in a given experiment. The issue becomes clear in linear processes where one can clearly separate the concept of the physical process from that of measurement or observation, such as it happens in conventional spectroscopy.

Typical cases in which that distinction is not at all clear appear in multiphoton excitation and dissociation of small polyatomic molecules induced by infrared laser radiation. ${ }^{2}$ In unimolecular absorption processes the concept of measurement is quantum in nature, and this implies that a measurement is inavoidably associated with a wave packet reduction ${ }^{1}$ where the observer and a classical measuring apparatus becomes necessarily involved. The question arises then where and what is the classical measuring apparatus in that case.

Unimolecular multiphoton dissociation (UMPD) processes offer a rare opportunity to deal with the above problem because here a typically quantum low-lying level region is smoothly coupled, via the quasicontinuum (QC), through the absorption mechanism, to a classical energy region where the molecule eventually dissociates. ${ }^{2}$ Thus, along the evolution of the vibrational molecular state, we may distinguish first a quantum region and then a classical one. The coupling between these two regions is not sufficient to induce any collapse of the wave packet, if it is governed by purely deterministic, unitary laws. For the collapse of the whole vibrational wave function to occur it is necessary an operating organizing principle which couples that wave function to just a sufficiently excited state belonging to a given mode. ${ }^{3}$ 
According to current ideas, the UMPD process takes place in a rather stochastic way. ${ }^{4}$ However, even if such an explanation would predict the observed results, it still could by no means be considered consistent as it provides with no explanation whatsoever for the reduction of the molecular wave function into a single-mode eigenstate.

Intramolecularly generated deterministic instabilities or chaos has been invoked in recent years as the fundamental mechanism which allows the multiphoton excitation to progress along the whole vibrational ladder. ${ }^{5,6}$ It now appears clearly that the effect of such an operating nonlinear process (which is thought to induce recurrence relations and feedback processes between the whole set of molecular background modes and particular modes of the same molecule) is twofold. On the one hand, it helps driving the molecule towards the uppermost excited molecular levels, and, on the other hand, it by itself supplies the required organizing principle leading to one particular mode excited up to the classical continuum. Thus, the induction of instabilities and chaos becomes a necessary ingredient for the UMPD to occur for any effective molecular size.

It follows that UMPD can be considered neither as a pure multiphoton excitation process nor as a purely measurement process, but it is partly a multiphoton process and partly a measurement process; i.e. some proportion of the absorbed infrared photons (at least those being absorbed by the first low-lying discrete levels according to pure quantum-mechanical rules) will mainly contribute the process, and the rest of them will be used by the whole system as probes to carry on the measurement of the dissociation process.

Hence we distinguish two different evolutions within UMPD. First, a unitary, deterministic evolution of the initial molecular vibrational state at time zero into a highly excited vibrational molecular state describable by a Schrödinger unitary time evolution operator. This evolution is time-symmetric, local and preserving coherence. At the same moment of the excitation process (typically when the level density of the background modes is high enough with respect to the wavelength of the driving radiation) one could situate the onset of a completely different evolution which is time-asymmetric, nonlocal and gives rise to a loss of relevant physical information (i.e. producing entropy). This evolution cannot be described by any quantummechanical evolution representation, but ought to follow the known rules of quantum measurements. We see thus that the UMPD exactly follows the two evolution regimes of conventional quantum mechanics (i.e. a unitary evolution and the ulterior measurement), with the "classical measuring apparatus" provided by the molecular system itself.

Since for UMPD all the molecules should be indistinguishable, when we irradiate a given sample, we actually are inducing a set of UMPD processes followed by their respective measurements. These processes and measurements will give then rise to a set of results subject to a quantum-mechanical probabilistic interpretation; i.e. all the possible dissociation channels should be switched on, each with the given probability, so that the final result will correspond to the quantum expectation value of, typically, the number of molecules being dissociated. 
2. Sufficiently small molecules oppose a strong bottleneck to absorption of infrared radiation. ${ }^{7,8}$ One should expect the rather narrow spectral region where the bottleneck operates to separate discrete from quasiclassical regions. In order to make that vibrational boundary apparent, one usually adds some inert gas which tends to fill rotational holes, so partly erasing the effect of that boundary. (See Figure 1.) The vibrational boundary may also be circumvented, and perhaps shifted, by irradiating the sample with two different infrared laser beams. The first infrared radiation resonates with a fundamental absorption of the molecule, while the second one, at

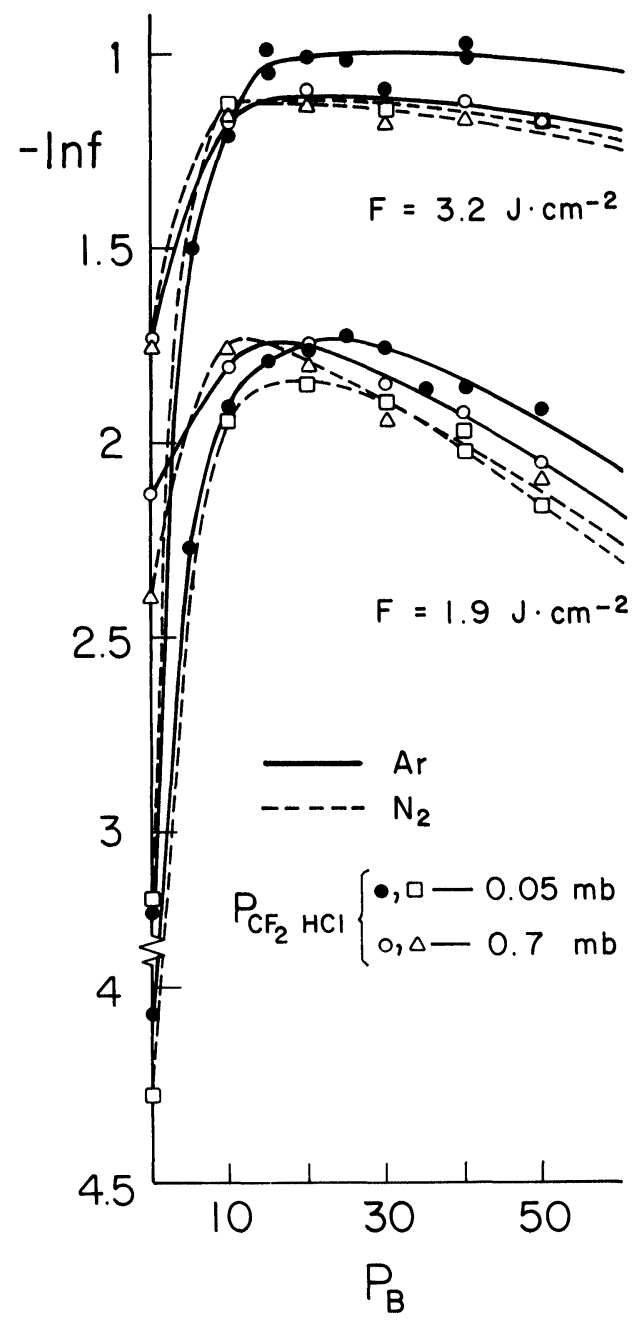

Figure 1 Deactivation of the bottleneck in MPD of $\mathrm{CF}_{2} \mathrm{HCl}$ as induced by increasing partial pressure, $P_{B}$, of the two buffers gases $\left(\mathrm{N}_{2}\right.$ and $\left.\mathrm{Ar}\right) . f$ is the fraction of molecules dissociated per laser pulse. ${ }^{9}$ 
a nonresonant wavelength, deactivates the bottleneck upwards. One thus expects the experiments using two wavelengths to be more effective and selective.

A question is, what happens with the generation of instabilities and chaos in two-wavelength experiments? At least at first glance, the greater effectiveness induced by the second nonresonant radiation should imply a more effective operating of the organizing principle. This would be made possible only when in the interaction process the two laser beams somehow cooperate, rendering the nature of the resulting absorption mechanism in some way equivalent to that in the single-wavelength process. A key point here is to identify and subsequently subtract the unphysical degrees of freedom in the whole matter-radiation system. These degrees of freedom largely manifest in the choice of the origin of energies in the Hamiltonian. When suitably gauged off, one is left with an interaction Hamiltonian where one can identify two new fundamental radiation modes associated with the center-of-frequency and relative-frequency of the two laser beams. ${ }^{6}$

A simplified theoretical model ${ }^{10}$ has been constructed using this Hamiltonian. It includes a reservoir where an infinite set of noninteracting background modes are involved, which couple to the molecular active mode and the induced two new radiation modes. Through this coupling mechanism recurrence phenomena are induced, leading to the generation of instabilities which, in turn, drive the high energy level absorption and eventually collapse the upper molecular vibration state into a given mode state in the continuum. The contribution of the active mode anharmonicity to instabilities is quite more effective in double irradiation than in single one. This explains why the former is more effective than the latter in what the dissociation yields is referred to (Figure 2).

3. The vibrational-induced cooperation between the two laser beams proceeds as follows. First, the two independent radiations are made to be correlated to each other through their nonlinear interaction with the matter system. Secondly, the molecule forces a double resonance process by which what actually interacts with the level system are the sum-of-frequency and relative-frequency radiation modes. These modes are coherently interacting with two corresponding molecular modes which are, at the same time, being intramolecularly generated out from the active mode and the reservoir.

In order to investigate the correlations between the two laser modes, we have carried out a series of double-wavelength experiments using $\mathrm{CF}_{2} \mathrm{HCl}$ as the molecular system, where we have introduced suitable delays between the two infrared laser beams. We have obtained (Figure 3) a sharp maximum in the dissociation yield when the delay between the two laser pulses is about $2.5 \mu$ s. This delay is fairly independent of the gas pressure.

The observed nonzero delay has been interpreted as being originated mainly from intramolecular vibrational energy redistribution which suitably stores the absorbed resonant radiation in a most accessible situation for the second nonresonant radiation to correlate with it. At the maximum of the curves of Figure 3 one should expect then the greatest correlation between the two absorbed radiations induced by the whole interaction process. 


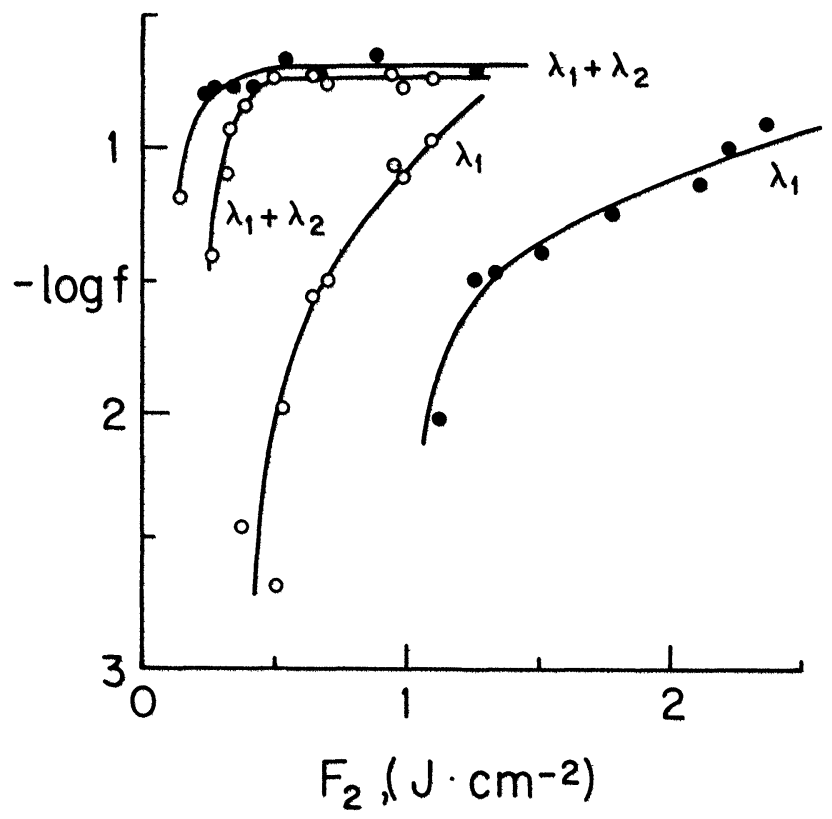

Figure 2 Fraction $f$ of $\mathrm{C}_{3} \mathrm{~F}_{6}(O)$ and $\mathrm{CF}_{2} \mathrm{HCl}(O)$ vs $\mathrm{F}_{1}$ for single $\left(\lambda_{1}\right)$ and double wavelength $\left(\lambda_{1}+\lambda_{2}\right)$ experiments. (O) $F_{2}=1.9 \mathrm{~J} \mathrm{~cm}-2, \lambda_{1}=9.603 \mu \mathrm{m}$ and $\lambda_{2}=10.148 \mu \mathrm{m}$; (O) $F_{2}=1.5 \mathrm{~J} \mathrm{~cm}^{-2}, \lambda_{1}=9.201 \mu \mathrm{m}$ and $\lambda_{2}=9.488 \mu \mathrm{m}$. In all cases $p=3$ mbar. ${ }^{11}$

After the system has reached the optimum resonant energy storing distribution, collisional deactivation begins taking place as it is shown by the nearly exponential character of the fall off of the curves in Figure 3 between 2.5 and $25 \mu \mathrm{s}$. At that point $V-T$ relaxation processes dominate as it was already pointed out by Borsella et al. ${ }^{13}$

The presence of a sharp peak in the curves of Figure 3 could also be interpreted by invoking a dependence of the process on intensity rather than fluence. ${ }^{14}$ However, defining intensity of the radiation in the case of double delayed exposure is not quite clear. When two equally lasting pulses illuminating the same sample volumen are exactly simultaneous, the intensity could be strictly defined as the sum of the two pulses energies divided by the time duration of just one of them. Nevertheless, if both pulses are delayed a time longer than their time width, the time for the joint intensity is not defined. Results of Figure 3 seems to suggest that a new delay dependent definition for double laser pulses is necessary. When the delay coincides with some given storage time for the resonant radiation, the correlation between the two beams, and hence also the yield of the dissociation process, becomes maximum. Thus, an effective single-shot notion can still be introduced for double irradiation. It will correspond to that single pulse, whose energy is the sum of the two individual pulse energies, which is associated with the optimum $\lambda_{1} / \lambda_{2}$ ratio and delay giving rise to a most effective dissociation, i.e. a maximum intramolecularly generated correlation between the two laser pulses. 


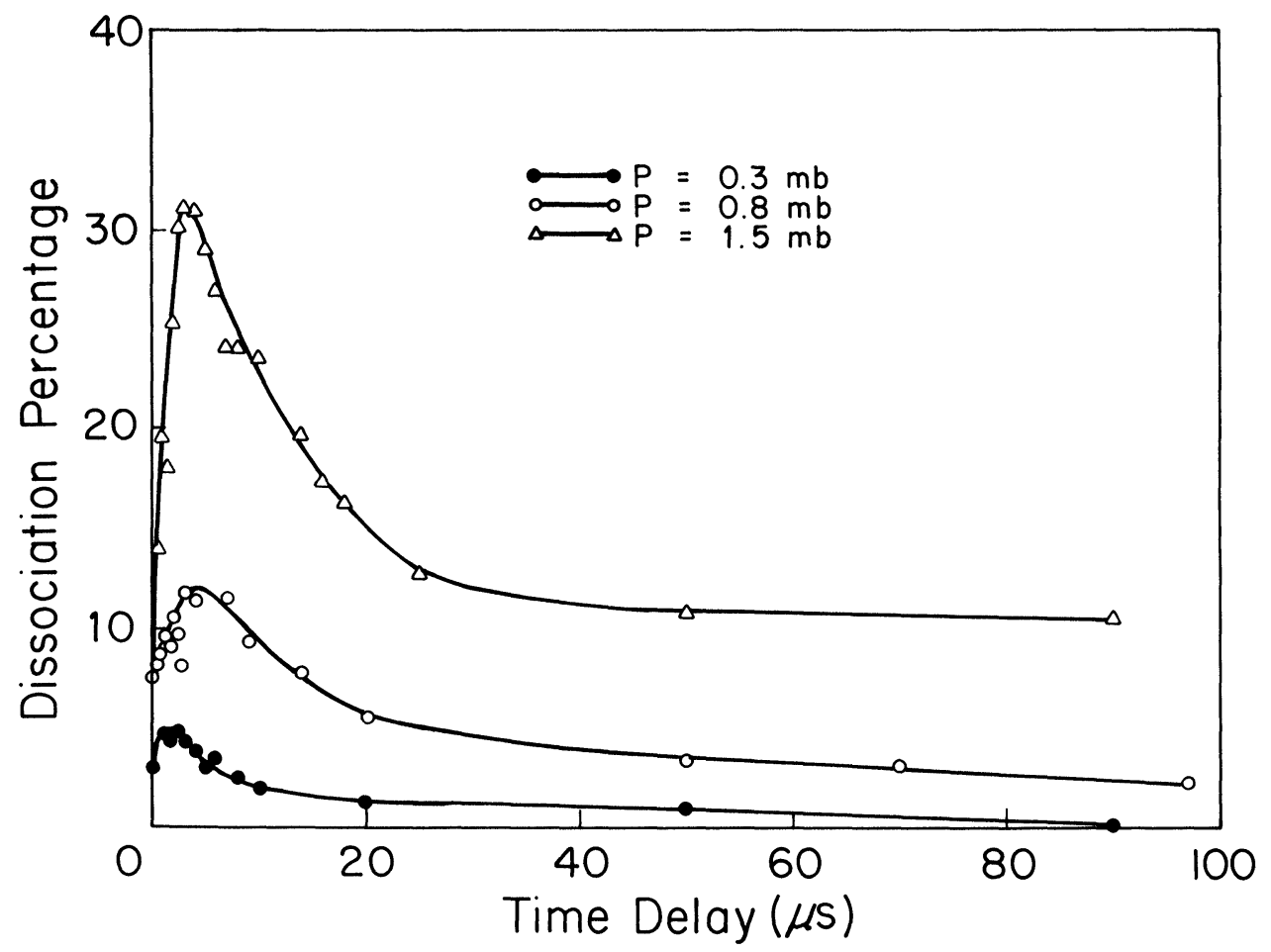

Figure 3 Dissociation probability (\%) of $\mathrm{CF}_{2} \mathrm{HCl}$ irradiated with two different wavelengths, $\lambda_{1}$ (resonant), $\lambda_{2}$ (nonresonant), $\left(\lambda_{1}=9.201 \mu \mathrm{m}, \lambda_{2}=9.488 \mu \mathrm{m} ; \phi_{1}=0.6 \mathrm{~J} \mathrm{~cm}^{-2}, \phi_{2}=1.7 \mathrm{~J} \mathrm{~cm}^{-2}\right.$, where $\phi$ denotes fluence) vs time delay between the two laser beams. ${ }^{12}$

\section{References}

1. J. A. Wheeler and W. H. Zurek, Quantum Theory of Measurement (Princeton Univ. Press, New Jersey, 1983).

2. C. D. Cantrell, Multiple-Photon Excitation and Dissociation of Polyatomic Molecules (Springer-Verlag, Berlin, 1986).

3. P. C. W. Davies, The Cosmic Blueprint (Unwin, London, 1987).

4. V. S. Letokhov, Progress in Optics XVI, edited by E. Wolf (North Holland, Amsterdam, 1978).

5. J. R. Ackerhalt and P. W. Milonni, Phys. Rev. A34, 1211 (1986).

6. C. L. Sigüenza, P. F. González-Díaz and M. Santos, Non Linear Dynamics and Quantum Phenomena, edited by R. Vilaseca and R. Corbalán, Springer Proceedings in Physics (Springer, Berlin, Heidelberg, 1991).

7. R. Duperrex and H. van den Bergh, J. Chem. Phys. 71, 3613 (1979).

8. M. Santos, C. Sigüenza, J. A. Torresano and P. F. González-Díaz, Spectrochim. Acta 46A, 455 (1990).

9. C. L. Sigüenza, Chem. Phys. Lett. 181, 351 (1990).

10. M. Santos, P. F. González-Díaz and C. L. Sigüenza. Talk delivered at the European Quantum Electronics conference, Edinburgh (UK), 1991. 
11. C. L. Sigüenza, M. Santos, J. A. Torresano, A. Pino and P. F. González-Díaz, Spectrochim. Acta 46A, 1499 (1990).

12. C. L. Sigüenza, M. Santos and P. F. González-Díaz (in preparation).

13. E. Borsella, R. Fantoni, G. Petrocelli, G. Sanna, M. Capitelli and M. Dilonardo, Chem. Phys. 63, 219 (1981).

14. E. Pochon, R. E. Weston, Jr. and G. W. Flynn, J. Phys. Chem. 89, 86 (1985). 